\title{
TRIATOMA SORDIDA - CONSIDERAÇÕES SOBRE O TEMPO DE VIDA DAS FORMAS ADULTAS E SOBRE A OVIPOSIÇÃO DAS FEMEAS*
}

José Maria Pacheco de Souza**

Vera Lúcia C. C. Rodrigues $* * *$

Eduardo Olavo da Rocha e Silva ****

\begin{abstract}
RSPUB $9 / 417$
SouzA, J. M. P. de et al. Triatoma sordida - Considerações sobre o tempo de vida das formas adultas e sobre a oviposição das fêmeas. Rev. Saúde públ., S. Paulo, 12:291-6, 1978.

RESUMO: Foram observados 53 exemplares do T. sordida que chegaram da fase alada, oriundos de 110 ovos; os machos eram em número de 22 e as fêmeas 31 . Os tempos médios de duração da fase de ninfa não foram estatisticamente diferentes entre os sexos, sendo 174,6 e 170,6 dias respectivamente para machos e fêmeas. O tempo médio de vida na fase alada foi significativamente maior para as fêmeas, com 503 dias para estas e 284 para os machos. $O$ periodo de oviposição correspondeu a $86,42 \%$ do tempo de fase alada das fêmeas, com a postura tendo maior intensidade na primeira metade desta fase. Cada fêmea pôs em média 570,8 ovos, variando a postura de 0,68 a 1,97 ovos por dia.
\end{abstract}

UNITERMOS: Triatomineos. Triatoma sordida. Tripanossomiase americana.

\section{I NTRODUCAO}

Observações relativas a aspectos do ciclo de vida dos triatomíneos, notadamente das espécies cujos exemplares invadem e colonizam nos ecótopos artificiais, interessam não somente à biologia e ecologia, como também às atividades de controle dos transmissores da doença de Chagas. Isso decorre das possiveis implicações para o homem da invasão, pelos triatomineos, das moradias e/ou construções anexas.
Fatos dessa natureza ocorrem com frequiência em regiōes do Estado de São Paulo, devido à presença de espécies ditas semidomiciliadas (Rocha e Silva e col. ${ }^{5}$, 1965), destacando-se entre elas o Triatoma sordida. Este triatomíneo apresenta ampla distribuição geográfica, sendo encontrado também nos estados do Piauí, Pernambuco, Bahia, Minas Gerais, Goiás, Mato Grosso, Paraná, Santa Catarina e Rio Grande do Sul. Fora do Brasil, sua presença já foi

* Apresentado no 140 Congresso da Sociedade Brasileira de Medicina Tropical e III Congresso da Sociedade Brasileira de Parasitologia, João Pessoa, PB, 1978.

* Do Departamento de Epidemiologia da Faculdade de Saúde Pública da USP - Av. Dr. Arnaldo, 715 - 01255 - Săo Paulo, SP - Brasil.

*** Da Superintendência de Controle de Endemias (SUCEN) da Secretaria da Saúde do Estado de São Paulo - Rua Tamandaré, $649-01525$ - São Paulo, SP — Brasil.

**** Do Departamento de Epidemiologia da Faculdade de Saúde Pública da USP e da SUCEN. 
SotZA, J. M. P. de et al. Triaioma sordida - Considerações sobre o tempo de vida das formas adultas e sobre a oviposição das fêmeas. Rev. Saúde públ., S. Paulo, 12:291-6, 1978.

assinalada na Bolívia, Paraguai, Argentina, Uruguai e Chile (Barretto ${ }^{1}$, 1968).

Apesar de sua ampla distribuição geográfica e marcante comportamento semidomiciliado, pelo menos em algumas áreas, são escassos, no entanto, estudos relativos ao ciclo de vida da espécie. Destacam-se, entre nós, o trabalho pioneiro de Pinto ${ }^{4}$ (1949) e mais recentemente, Forattini e col. ${ }^{3}$ (1977) que acompanharam o desenvolvimento do $T$. sordida, de ovo a alado, em colônias espontaneamente mantidas em galinheiros experimentais, no campo.

Devido ao comportamento peculiar das formas aladas que buscam novos abrigos (fenômeno da dispersão), pouco puderam relatar sobre o tempo de vida dos exemplares da espécie na fase adulta e sobre a oviposição das fêmeas. As dificuldades para este tipo de experimento são inerentes ao tipo de trabalho realizado por aqueles autores, ou seja, observação de campo. Em virtude disto, considerou-se de interesse a efetivação de um experimento, em laboratório, que abordasse aspectos da vida do $T$. sordida, no caso, somente dos exempla" res que atingem a fase alada.

Em particulr, decidiu-se estudar o tempo de vida de exemplares da espécie, como ninfa e alado, as possiveis correlações entre estes tempos e, para fêmeas, também as variáveis referentes à oviposição, quais sejam, duração e número de ovos.

\section{MATERIAI E METODOS}

Foram observados 53 exemplares que chegaram à fase alada, oriundos de um lote de 110 ovos, referentes à postura de três dias consecutivos de $\mathbf{5 5}$ fêmeas. Estas, haviam sido capturadas em junho de 1974, em localidades rurais da região de Ribeirão Preto.

O experimento foi conduzido no antigo Insetário da SUCEN, em Moji Guaçu, Estado de São Paulo, com temperatura ambiente em torno de $25-28^{\circ} \mathrm{C}$ e com umidade relativa do ar oscilando de 60 a $80 \%$.
Os exemplares foram colonizados em lotes, de acordo com o estádio; na medida em que se processava seu ciclo evolutivo, o número de insetos em cada lote diminuia, até que, ao atingir o $5^{\circ}$ estádio, cada espécime passou a ser mantido isoladamente, em um frasco de Borrel de $9 \mathrm{~cm}$ de altura e $4 \mathrm{~cm}$ de diâmetro. O fundo do Borrel era forrado com papel de filtro, periodicamente trocado e tinha a boca vedada por filó de náilon, firmemente fixado na posição por elásticos. No interior de cada frasco foi colocado um suporte de cartolina branca de $9 \times 3 \mathrm{~cm}$, dobrado em $\mathrm{V}$, para facilitar o deslocamento do exemplar em busca do alimento. Os repastos sanguíneos foram realizados, durante todo o processo de desenvolvimento dos triatomíneos, em camundongos albinos jovens, de oito em oito dias, durante aproximadamente $45 \mathrm{~min}$. de cada vez.

Ao atingir a fase alada, cada exemplar passou a ser diariamente observado, à exceção dos domingos, e os dados colhidos foram anotados em caderneta própria. Para cada inseto foram anotados os tempos de vida como ninfa e alado, sendo esta última fase subdividida, no caso das fêmeas, em períodos: ante-oviposição, oviposição e pósoviposição. Ainda para as fêmeas, obtevese o número de ovos de cada uma.

Os resultados são descritos sob a forma de médias aritméticas, desvios padrão, medianas e coeficientes de correlação de Pearson. Os testes estatísticos foram o de Mann - Whitney para diferenças de médias e da transformação logaritmica do coeficiente de correlação de Spearman para diferenças entre correlação (Costa Neto ${ }^{2}$, 1977); o nível de significância escolhido foi $5 \%$, sendo apresentada também a significância descritiva $(P)$ em cada teste.

\section{RESULTADOS E COMENTARIOS}

Dos 110 ovos inicialmente disponíveis, atingiram a fase alada 53 triatomíneos $(48,18 \%)$, sendo 22 exemplares do sexe 
SouzA, J. M. P. de et al. Triatoma sordida - Consideraçōes sobre o tempo de vida das formas adultas e sobre a oviposição das fêmeas. Rev. Saúde públ., S. Paulo, 12:291-6, 1978.

masculino e 31 do sexo feminino. As tabelas 1 e 3 apresentam os dados referentes ao tempo de vida de cada exemplar. $\mathrm{O}$ macho de vida mais longa atingiu 788 dias, dos quais 241 como ninfa e 547 como adulto. A fêmea de vida maior atingiu 244 dias como ninfa e 719 como imago, num total de 963 dias.

A comparação dos tempos médios de vida entre os sexos chama de imediato a atenção pois, enquanto os tempos de duração da fase de ninfa são praticamente iguais, o tempo de vida como alado é quase duas vezes maior para as fêmeas. $O$ teste de Mann-Whitney corrobora estes achados; os valores de $z$ foram, respectivamente, 1,16 $(P=0,12)$ e $3,85(P=0,00006)$.
Nos machos, a correlação de Pearson entre tempos como ninfa e alado foi 0,029 e nas fêmeas $-0,076$. As correlações de Spearman, utilizadas no teste de comparação, foram de 0,026 e $-0,210$; após as devidas transformações logaritmicas, o teste de diferença não mostrou significância, com $z=0,80(P=0,21)$.

As comparações acima permitem inferir que a diferença fundamental existente no tempo de vida dos animais que chegam até à forma alada está ligada à atividade de procriação. A fêmea dispõe de vida mais longa, no sentido de aumentar a probabilidade de preservação da espécie, através de seu prolongado tempo de oviposição. Esta suposição, talvez óbvia para alguns, é re-

TABELA 1

Dias de vida em condições de laboratório (fases de ninfa e alaía), de 22 exemplares machos do T. sordida. Moji Guaçu, $1974-1977$.

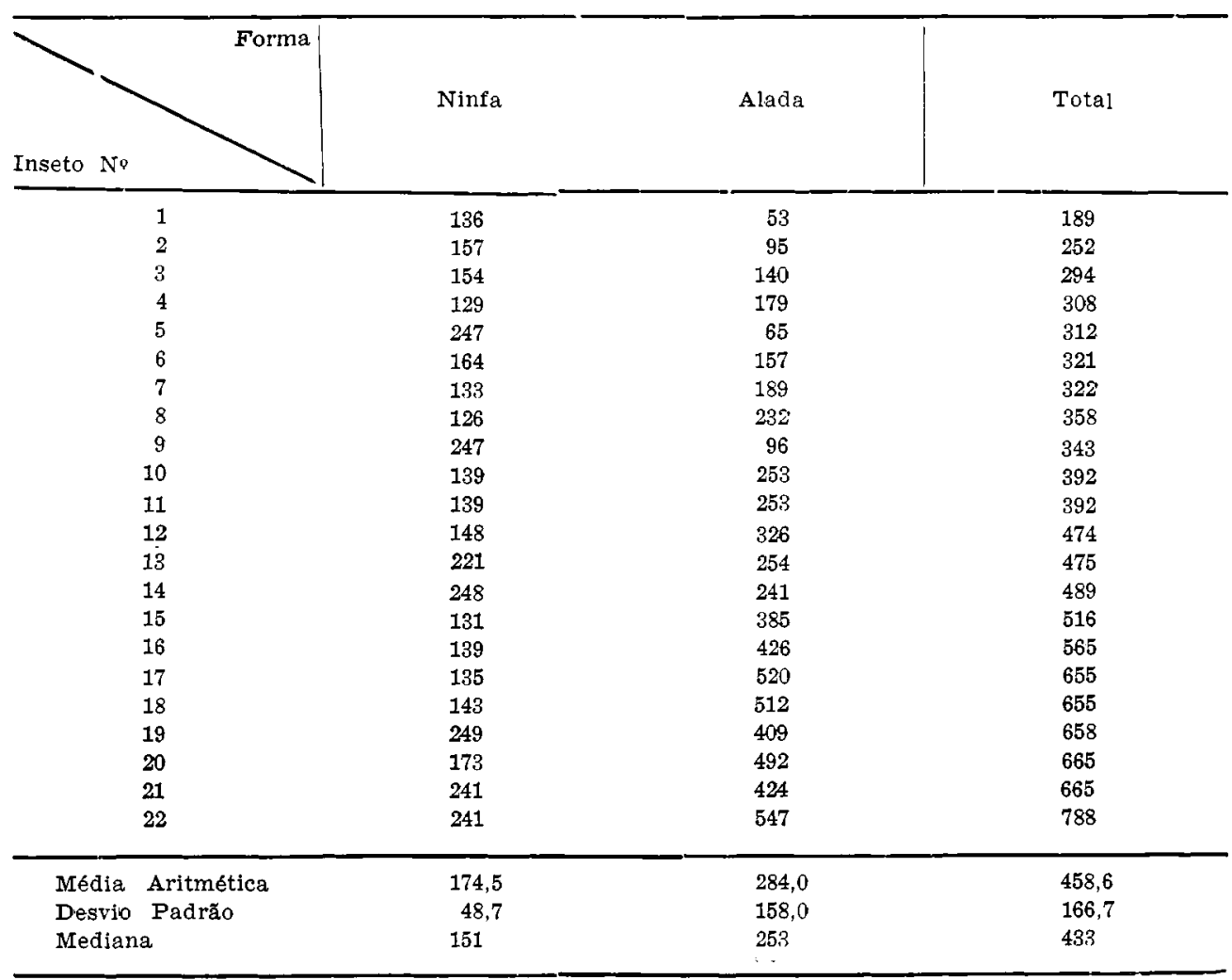


SoUzA, J. M. P. de et al. Triatoma sordida - Considerações sobre o tempo de vida das formas adultas e sobre a oviposição das fêmeas. Rev. Saúde públ., S. Paulo, 12:291-6, 1978.

T A B E L A 2

Dias de vida em condições de laboratório (fases de ninfa e alada), de 31 exemplares fêmeas do T. sordida. Moji Guaçu, 1974 - 1977.

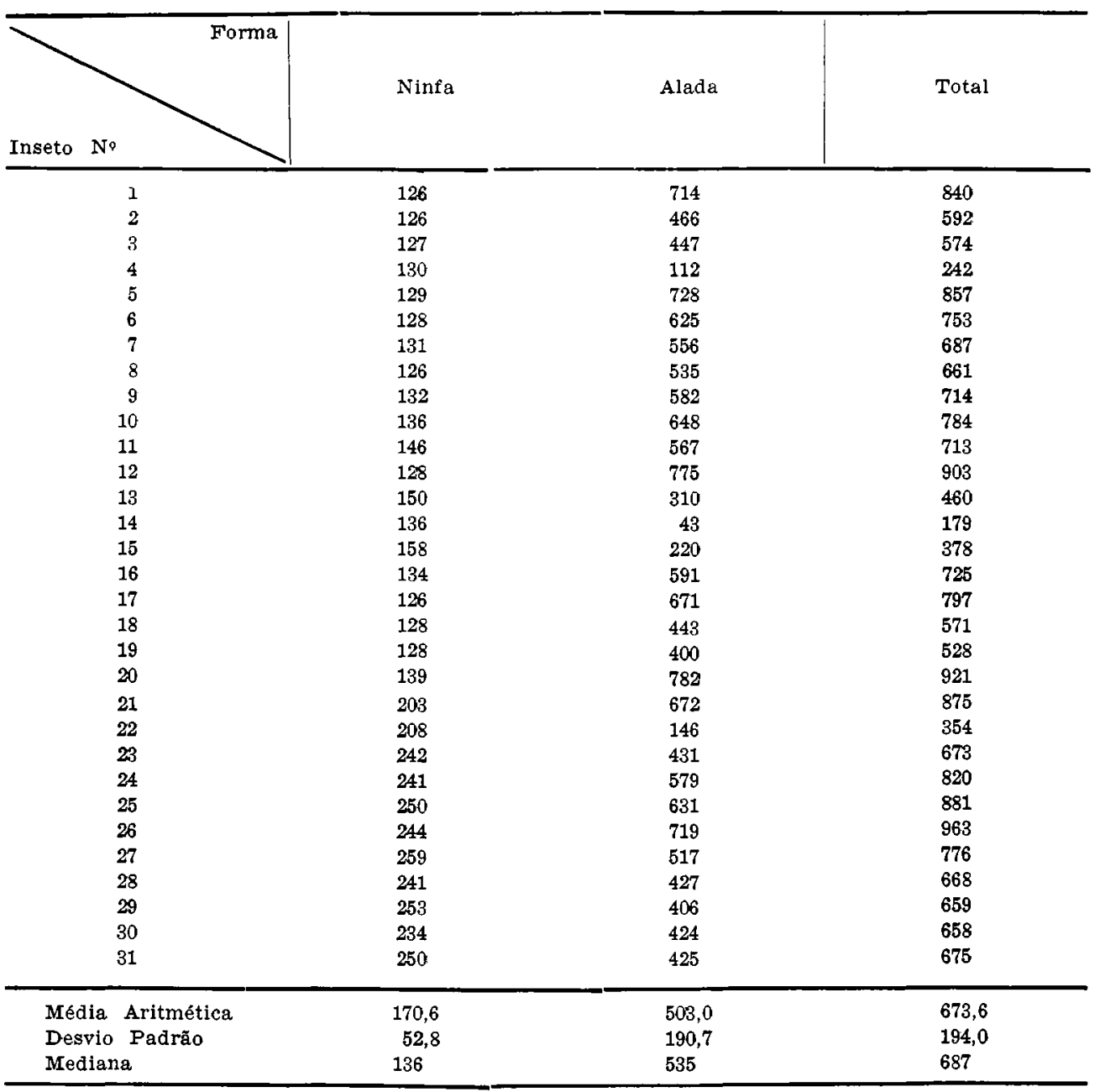

forçada pelo fato de $86,42 \%$ do tempo de duração média da fase alada das fêmeas corresponder ao período de oviposição, período este medido em dias decorridos entre a primeira e última postura.

A Tabela 3 apresenta resultados corres pondentes somente às fêmeas, em sua fase adulta. O maior tempo de oviposição foi
693 dias e o menor foi zero, este último pela postura ter durado menos de 24 horas; o segundo menor tempo foi 68 dias. 0 número de ovos variou de um mínimo de 3 a um máximo de 1.281 , ficando o número médio de ovos postos por dia entre 0,68 e 1,97, excluindo-se o espécime número 14 , pois o denominador é zero. 
SOUZA, J. M. P. de et al. Triatoma sordida - Considerações sobre o tempo de vida das formas adultas e sobre a oviposição das lêmeas. Rev. Saúde públ., S. Paulo, 12:291-6, 1978.

TABELA 3

Duração do periodo de ante-oviposição, oviposição, pós-oviposição, número de ovos postos e número médio de ovos/dia, de 31 exemplares fêmeas de $T$. sordida, observados em laboratório. Moji Guaçu, 1974 - 1977.

\begin{tabular}{|c|c|c|c|c|c|}
\hline Fase & Ante-oviposição & Oviposição & Pós-Oviposição & $\begin{array}{l}\text { Ovos } \\
\text { postos }\end{array}$ & $\begin{array}{c}\text { Ovos/ } \\
\text { Dia }\end{array}$ \\
\hline 1 & 22 & 686 & 6 & 1.281 & 1,87 \\
\hline 2 & 26 & 426 & 14 & 421 & 0,99 \\
\hline 3 & 30 & 410 & 7 & 486 & 1,19 \\
\hline 4 & 26 & 68 & 18 & 109 & 1,60 \\
\hline 5 & 32 & 693 & 3 & 918 & 1,32 \\
\hline 6 & 33 & 585 & 7 & 775 & 1,32 \\
\hline 7 & 33 & 490 & 33 & 728 & 1,49 \\
\hline 8 & 38 & 494 & 3 & 723 & 1,46 \\
\hline 9 & 36 & 543 & 3 & 781 & 1,44 \\
\hline 10 & 32 & 539 & 77 & 464 & 0,86 \\
\hline 11 & 23 & 541 & 3 & 644 & 1,19 \\
\hline 12 & 42 & 582 & 151 & 520 & 0,89 \\
\hline 13 & 22 & 281 & 7 & 478 & 1,70 \\
\hline 14 & 37 & - & 6 & 3 & $* *$ \\
\hline 15 & 20 & 194 & 6 & 287 & 1,48 \\
\hline 16 & 48 & 539 & 4 & 518 & 0,96 \\
\hline 17 & 60 & 554 & 57 & 565 & 1,02 \\
\hline 18 & 61 & 355 & 27 & 253 & 0,71 \\
\hline 19 & 85 & 313 & 2 & 213 & 0,68 \\
\hline 20 & 74 & 548 & 160 & 862 & 1,57 \\
\hline 21 & 21 & 521 & 130 & 628 & 1,21 \\
\hline 22 & 24 & 120 & 2 & 171 & 1,43 \\
\hline 23 & 17 & 410 & 4 & 809 & 1,97 \\
\hline 24 & 25 & 551 & 3 & 1.010 & 1,83 \\
\hline 25 & 20 & 595 & 16 & 962 & 1,62 \\
\hline 26 & 32 & 669 & 18 & 1.111 & 1,66 \\
\hline 27 & 17 & 382 & 118 & 373 & 0,98 \\
\hline 28 & 37 & 369 & 21 & 392 & 1,06 \\
\hline 29 & 31 & 371 & 4 & 608 & 1,64 \\
\hline 30 & 52 & 368 & 4 & 342 & 0,93 \\
\hline 31 & 43 & 278 & 104 & 260 & 0,94 \\
\hline Média Aritmética & 35,5 & 434,7 & 32,8 & 570,8 & $1,31^{*}$ \\
\hline Desvio Padrão & 16,5 & 175,2 & 48,1 & 307,0 & $* *$ \\
\hline Mediana & 32 & 490 & 7 & 520 & 1,32 \\
\hline
\end{tabular}

* $1,31=570,8 \div 434,7$

** năo calculado

Houve também interesse em verificar se a intensidade da postura foi uniforme, no decorrer de todo o tempo de oviposição. Excluindo-se a fêmea 14 da análise, observou-se que 25 dos 30 insetos eliminaram mais de $50 \%$ do seu total de ovos, antes de completar $50 \%$ de seu período de ovi- posição, ou seja, a postura é mais intensa no início do periodo. Valendo ressaltar ainda que as posturas mínima e máxima observadas respectivamente de 1 ovo e 18 ovos, postos em um só dia.

Os resultados evidenciam atuarem as fêmeas como verdadeiras "poedeiras", dis- 
SOUZA, J. M. P. de et al. Triatoma sordida - Considerações sobre o tempo de vida das formas adultas e sobre a oviposição das fèmeas. Rev. Saúde públ., S. Paulo, 12:291-6, 1978.

persando uma média de 570,8 ovos cada, durante periodo superior a um ano, fato de relevante valor na manutenção da espécie, pois em periodo tão amplo haverá certamente diversas épocas favoráveis à viabilização de certo número de ovos.

\section{CONSIDERAÇOES FINAIS}

Os resultados aqui obtidos não se aplicam diretamente ao campo, onde as condiçōes são obviamente diferentes daquelas presentes em um insetário. Não deixam, no entretanto, de serem úteis como primeira aproximação, permitindo, inclusive, aprimorar conhecimentos que possam levar de algum modo à melhoria das atividades de controle dos vetores da doença de Chagas, notadamente nas áreas limpas do $T$. infestans, mas com problemas de invasão dos ecótopos artificiais por espécies semidomiciliadas, como a estudada.

\section{AGRADECIMENTO}

Ao Dr. Octávio Alves Ferreira, da Superintendência de Controle de Endemias (SUCEN), pela gentileza em ceder o material utilizado.

SouzA, J. M. P. de et al. [Triatoma sordida: Comments on the life span of the adult forms and oviposition of females] Rev. Saúde públ., S. Paulo, 12: 291-6, 1978.

ABSTRACT: Fifty three specimen of $\mathrm{T}$. sordida which arrived at the adult stage were studied; there were 22 males and 31 females, all obtained from 110 eggs. The average time duration of life in the nymph phase was not statistically different between sexes, being 174.6 and 170.6 days respectively for mate and female. The females had a larger mean time duration of life as adults (503 days) than the males (284 days). During their adult life, $86.42 \%$ of the time corresponded to oviposition, the greater intensity of egg-laying ocurring during the first half of the period. The mean number of eggs was 570.8 and the ratio egg/day varied from 0.68 to 1.97 .

UNITERMS: Triatoma sordida. Trypanosomiasis, South American.

\section{REFERENCIAS BIBLIOGRAFICAS}

1. BARRETTO, M. P. Transmissores do Triatoma cruai - os triatomineos. In: Cançado. R. et al., ed. Doença de Chagas. Belo Horizonte, 1968. p. 189224.

2. COSTA NETO, P. L. de O. Estatistica. São Paulo, Ed. Edgard Blücher, 1977.

3. Forattini, O. P. et al. Aspectos ecologicos da Tripanossomíase americana. $\mathrm{X}$-Dados populacionais das colônias de Panstrongylus megistus e de Triatoma sordida espontaneamente desenvolvidas em ecótopos artificiais. Rev. Saude públ., S. Paulo, 11:362-74, 1977.
4. PINTO, C. F. Fatos curiosos sobre a biologia do Triatoma sordida (Nota prévia) Rev. bras, Med., 6:305, 1949.

5. ROCHA E SILVA, E. O. da et al. Suspensão do rociado no combate ao Triatoma infestans em áreas do Estado de São Paulo, Brasil. Rev. Saúde públ., S. Paulo, 3:173-81, 1969.

Recebido para publicasão em 22/11/197\% Aprovado para publicasão em 13/04/1978 